\title{
RadioPropa - \\ A Modular Raytracer for In-Matter Radio Propagation
}

\author{
Tobias Winchen ${ }^{1, \star}$ \\ ${ }^{1}$ Astrophysical Institute, Vrije Universiteit Brussel, Pleinlaan 2, 1050 Brussels, Belgium
}

\begin{abstract}
Experiments for radio detection of UHE particles such as e.g. ARA/ARIANNA or NuMoon require detailed understanding of the propagation of radio waves in the surrounding matter. The index of refraction in e.g. polar ice or lunar rock may have a complex spatial structure that makes detailed simulations of the radio propagation necessary to design the respective experiments and analyse their data. Here, we present RadioPropa as a new modular ray tracing code that solves the eikonal equation with a Runge-Kutta method in arbitrary refractivity fields. RadioPropa is based on the cosmic ray propagation code CRPropa, which has been forked to allow efficient incorporation of the required data structures for ray tracing while retaining its modular design. This allows for the setup of versatile simulation geometries as well as the easy inclusion of additional physical effects such as e.g. partial reflection on boundary layers in the simulations. We discuss the principal design of the code as well as its performance in example applications.
\end{abstract}

\section{Introduction}

Several experiments such as e.g. ARA [1], ARIANNA [2] or NuMoon [3] are currently in preparation that use the radio emission by the Askaryan effect [4] of a particle shower developing in matter to detect high energetic cosmic particles. Design of these experiments and also future interpretation of the collected data requires detailed simulation of the propagation of the radio pulses from the interaction point to the receiving antennas. Simulations of the wave propagation in full detail with the finite-differences-time-domain (FDTD) technique [5] are too time consuming to be used for all tasks. Faster solutions such as ray-tracing or analytical estimates are thus required, that can simulate versatile geometries as well as arbitrary models for the refractive index.

While ray-tracing can, in principle, simulate arbitrary geometries and models for the refractive index, the underlying approximation of geometrical optics does not include all relevant effects. To achieve the necessary detail nevertheless, shortcomings in the ray-tracing may be included by additional parametrizations tuned to full FDTD simulations. This will require a dedicated modular code for the task and disfavours using available ray-tracing codes.

As basis for such a new code I use here the CRPropa software [6] that has been originally designed for the simulation of high energetic cosmic rays. The highly modular software framework is easily extendable and already provides several necessary features such as boundary conditions, data container, file-IO, geometry descriptions etc. - only the required physics modules for the propagation

^e-mail: tobias.winchen@ rwth-aachen.de; This work is funded by ERC grant 640130 and DFG grant WI 4946/1-1 
of rays have to be created and some trivial changes to the central data structure have to be included to trace rays with this software.

\section{Propagator for Gradient Media and Handling of Media Boundaries}

The path of a ray $\mathbf{r}(s)$ with path parameter $s$ in a medium with index of refraction $n(\mathbf{r})$ is described by the eikonal equation

$$
\frac{\mathrm{d}}{\mathrm{d} s}\left(n(\mathbf{r}) \frac{\mathrm{d} \mathbf{r}}{\mathrm{d} s}\right)=\nabla n
$$

which is in general difficult to solve. In the modular software presented here, boundary effects are treated completely separate from propagation in gradient-index media. This allows to use a locally paraxial approximation for the latter, i.e. assuming that in any individual step of the algorithm the change of the refractive index along the path $d s$ is small. Consequently eq. 1 simplifies to

$$
\nabla n \approx n(\mathbf{r}) \frac{\mathrm{d}^{2} \mathbf{r}}{\mathrm{d} s^{2}}
$$

which can be easily solved. For this purpose, I adapted the Cash-Karp solver [7] already implemented in CRPropa.

Traversing a boundary, the ray is reflected and eventually refracted according to Snell's law. Due to the paraxial-approximation discussed above, such boundary conditions are treated in a separate module and consequently have to be defined explicitly in the setup of the simulation. If the ray passes a boundary, the ray is reflected and a secondary ray is eventually cast from the intersection point. The amplitudes of the rays are determined according Fresnell's equations.

In figure 1 an example simulation for in-ice radio propagation demonstrating these features is shown. The left part of the figures shows the ray traces, the right part the profile of the refractive index. The model for the refractive index is $n(z)=n_{0}-\Delta n e^{-z / z_{0}}$ with $n_{0}=1.788, \Delta n=0.463$ and $z_{0}=71.4 \mathrm{~m}$, below the ice-air boundary at $z=0 \mathrm{~m}$ with an additional layer of higher refractive index $n_{1}=1.5$ from $0 \mathrm{~m}$ to $10 \mathrm{~m}$. Rays are emitted in several directions from a point in $240 \mathrm{~m}$ depth. The rays are bent in the gradient media and reflected, respectively refracted, at the boundary surfaces as expected. Rays are followed to a maximum propagation time of the ray of $5 \mu \mathrm{s}$ and rays with an amplitude $A$ below the initial amplitude $A_{0}$ of $A / A_{0}<0.05$ are discarded.

In this example all rays are emitted in the $\mathrm{X}-\mathrm{Z}$ plane for presentation clarity only, the simulation is in three dimensions. Versatile simulation setups with e.g. distributions of the refractive index from gridded data or arbitrary equations, receiver/emitter locations, and boundary shapes as in particular curved boundaries are also already possible with the software.

The accuracy of the simulation is determined by the paraxial-approximation of the eikonal, the minimum step-length and tolerance of the integration. The latter two are both free parameters of the simulation that are limited only by the invested computing time. To investigate the accuracy I compared the result of simulations with analytical solutions for gradient-index media with $n(z)=$ $n_{0}-\Delta n e^{-z / z_{0}}$ for $\mathrm{z}<0$ and $n=1$ for $z>0$ and parameters as above. I traced rays with various launch angles up to a linear distance of $1500 \mathrm{~m}$ from the source and compared the parameters with the analytical solution at the observation point. The launch angles agree in this scenario to better than $0.01^{\circ}$, the path length to better than $0.005 \mathrm{~m}$ and the travel time to better than $7 \times 10^{-12} \mathrm{~s}$.

\section{Conclusion}

I demonstrated that the cosmic ray propagation code CRPropa is a solid base for a radio propagation code requiring only few modifications, that, however, still justify a fork of the code. The resulting 


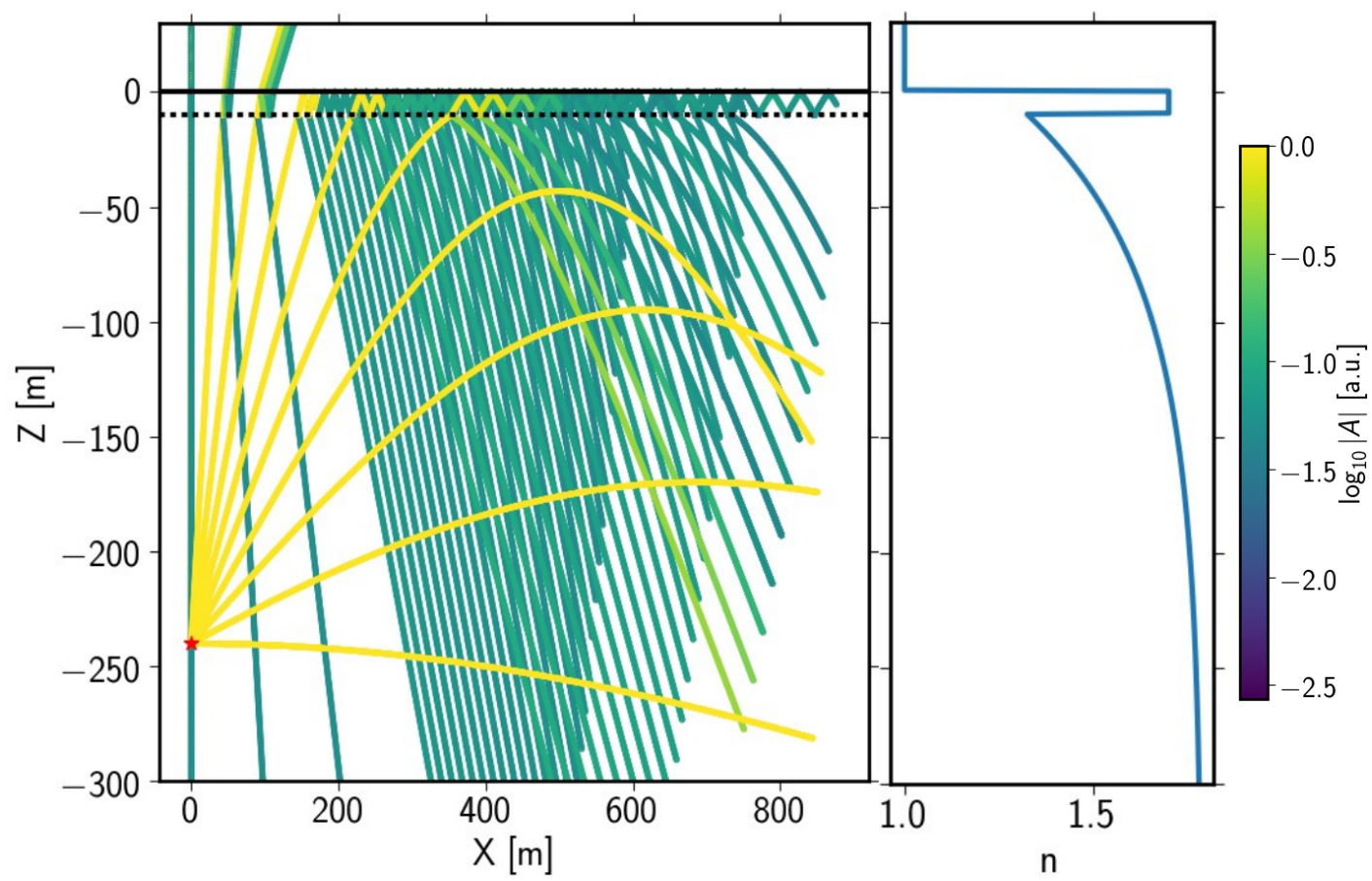

Figure 1. Example of propagated rays (left) in a two layer ice-model with varying index of refraction $n$ in $\mathrm{z}$ direction (right). Colors denote the amplitude of the ray. Correction of the amplitude for length of the ray path is not included here to emphasize the effect of multiple reflection.

code 'RadioPropa' is a modular code for in matter ray tracing that handles boundary conditions and gradient-index media with a local paraxial approximation. Due to the modular structure, alternative propagators with higher accuracy and parametrizations for additional effects can be easily included if needed in the future. RadioPropa is free software published under the GPLv3 license and can be downloaded from https://github.com/TobiasWinchen/RadioPropa.

\section{References}

[1] P. Allison et al., Astroparticle Physics 35, 457 (2012)

[2] A. Hallgren for the ARIANNA Collaboration, EPJ Web of Conferences 116, 03003

(2016)

[3] T. Winchen et al. in this proceedings

[4] G. Askaryan, Sovjet Physics J.E.T.P 14, 441 (1962)

[5] K. Yee, IEEE Transactions on Antennas and Propagation 14, 302 (1966)

[6] R. Alves Batista et al., JCAP 05, 038 (2016)

[7] J.R. Cash, A.H. Karp, ACM Trans. Math. Softw. 16, 201 (1990) 